\title{
Field Algebras do not Leave Field Domains Invariant
}

\author{
S. S. Horuzhy and A. V. Voronin \\ Steklov Mathematical Institute, SU-117333 Moscow, GSP-1, USSR
}

\begin{abstract}
It is proved that von Neumann algebras associated to Op*-algebra $(\mathscr{P}, \mathscr{D})$ cannot leave the domain $\mathscr{D}$ of $\mathscr{P}$ invariant if they are type I or type III factors or finite direct sums of such factors. Hence it follows that in quantum field theory global and local von Neumann field algebras in typical cases do not leave invariant the definition domain of Wightman fields.
\end{abstract}

In the theory of Op*-algebras it is often necessary or just helpful to consider von Neumann algebras associated in various ways to a given Op*-algebra $(\mathscr{P}, \mathscr{D})$. E.g., one of such algebras is $\left(\mathscr{P}_{w}^{\prime}\right)^{\prime}$, where $\mathscr{P}_{w}^{\prime}$ is the weak bounded commutant of $\mathscr{P}$, and there are many others (cf. [1, Sect. 8.1]) which are usually larger because $\mathscr{P}_{w}^{\prime}$ is the largest of all bounded commutants of $\mathscr{P}$. We are going to demonstrate now that there is strong connection between the structure of these von Neumann algebras and their property to leave or not to leave invariant the domain $\mathscr{D}$ of $\mathscr{P}$. The result is in itself quite simple but it has interesting implications for quantum field theory: it turns out that field $W^{*}$-algebras, global as well as local, under rather general conditions cannot leave invariant the definition domain of field operators (Gårding domain of Wightman theory). We also get some restrictions on admissible classes of field Op*-algebras.

We call Op*-algebra $(\mathscr{P}, \mathscr{D})$ nontrivial if it does not coincide with its bounded part, $\mathscr{P} \neq \mathscr{P}_{b}=\mathscr{P} \cap \mathfrak{B}(\mathscr{H})$, and we denote the involution in $\mathscr{P}$ as $A^{\dagger}=\left.A^{*}\right|_{\mathscr{D}}, A \in \mathscr{P}$, and the strong bounded commutant of $\mathscr{P}$ as $\mathscr{P}_{s}^{\prime}$. Our main statement is the following

Theorem. Let $(\mathscr{P}, \mathscr{D})$ be a nontrivial Op*-algebra such that $\mathscr{P}_{s}^{\prime}=\mathscr{P}_{w}^{\prime}$ or including at least one essentially self-adjoint unbounded operator, and let $R$ be a von Neumann algebra such that $R \mathscr{D} \subset \mathscr{D}$ and $R \supset\left(\mathscr{P}_{w}^{\prime}\right)^{\prime}$. Then $R$ is not a type I or type III factor. Moreover, if $\mathscr{P}_{s}^{\prime}=\mathscr{P}_{w}^{\prime}$ or $\mathscr{P}$ is generated by a system of essentially self-adjoint operators, then $R$ is not a finite direct sum of type I and/or type III factors.

Proof. Let us show that $R$ includes spectral projections of a self-adjoint extension $\tilde{A}$ of some unbounded operator $A \in \mathscr{P}$. In the case $\mathscr{P} \ni A, \bar{A}=A^{*}, A \notin \mathfrak{B}(\mathscr{H})$, let us denote $E(\lambda)$ the spectral measure of $\tilde{A} \equiv \bar{A}$. It is easy to check that any operator $B \in \mathscr{P}_{w}^{\prime}$ commutes weakly with $\bar{A}$ and leaves its domain invariant. Hence, $B$ commutes strongly with $\bar{A}$ and, by the spectral theorem, commutes with $E(\lambda)$, so we get $E(\lambda) \in\left(\mathscr{P}_{w}^{\prime}\right)^{\prime}$. In the general case, for $A_{1} \in \mathscr{P}, A_{1} \notin \mathfrak{B}(\mathscr{H})$, let us denote $\tilde{A}$ the Friedrichs' extension of the positive unbounded operator $A=A_{1}^{\dagger} A_{1} \in \mathscr{P}$ and $E(\lambda)$ the spectral measure of $\tilde{A}$. A unitary operator commuting strongly with $A$ commutes strongly with $\tilde{A}[2$, p. 358$]$ and therefore it commutes with $E(\lambda)$. Hence 
$E(\lambda)$ commutes with all unitary operators of $\mathscr{P}_{s}^{\prime}$, and if $\mathscr{P}_{s}^{\prime}=\mathscr{P}_{w}^{\prime}$, we get again $E(\lambda) \in\left(\mathscr{P}_{w}^{\prime}\right)^{\prime}$.

Now we can suppose without restriction that $E(\lambda) \neq I$ for all $\lambda>0$ and choose an infinite sequence $\left\{\alpha_{n}\right\}$ of positive numbers such that

$$
E\left(\alpha_{n}+1\right) \neq E\left(\alpha_{n}\right) ; \quad \alpha_{1}>0, \quad \alpha_{n+1} \geqq \alpha_{n}+3 n^{2}, \quad n=1,2, \ldots .
$$

Let us suppose also that $R$ is a type I or III factor and notice that in this case for the infinite sequence of nonzero projections $E_{n}=E\left(\alpha_{n}+1\right)-E\left(\alpha_{n}\right)$ in $R$ there exists a sequence of their subprojections $F_{n}$ which also belong to $R$ and are all mutually equivalent, i.e.

$$
F_{n} \in R, \quad 0 \neq F_{n} \leqq E_{n}, \quad F_{n} \sim F_{m} \bmod R .
$$

Indeed, if $R$ is a discrete factor, then every $E_{n}$ majorizes some minimal projection $F_{n}$ from $R$ and all minimal projections are equivalent; if $R$ is a type III factor, then every $E_{n}$ majorizes some $\sigma$-finite projection $F_{n}$ from $R$ and all such projections are equivalent [3, p. 299]. Due to (2), there are partial isometries $T_{n}$ such that

$$
T_{n} \in R, \quad T_{n}^{*} T_{n}=F_{1}, \quad T_{n} T_{n}^{*}=F_{n}, \quad n=1,2, \ldots .
$$

If we now consider the series $\sum_{n=1}^{\infty} \frac{1}{\sqrt{\alpha_{n}}} T_{n}^{*}$, then taking into account that

we get from (1) the estimate

$$
\lim _{k \rightarrow \infty}\left(3 \sum_{n=1}^{k} n^{2} / k^{3}\right)=1
$$

$$
\left\|\frac{1}{\sqrt{\alpha_{n}}} T_{n}^{*}\right\| \leqq C n^{-3 / 2} \text { at } n \rightarrow \infty .
$$

It implies that the series is uniformly convergent and defines the operator $B=\sum_{n=1}^{\infty} \frac{1}{\sqrt{\alpha_{n}}} T_{n}^{*}$ belonging to $R$.

We shall prove that the unbounded operator $X=B A$ is not closable. To this end we construct a sequence of vectors $\Phi_{n} \in \operatorname{dom} X=\mathscr{D}$ such that $\lim _{n \rightarrow \infty} \Phi_{n}=0$, but $\lim _{n \rightarrow \infty} X \Phi_{n} \neq 0$. Let us take an arbitrary nonzero vector $\Phi_{1} \in F_{1} \mathscr{D}$, and put

$$
\Phi_{n}=\frac{1}{\sqrt{\alpha_{n}}} T_{n} \Phi_{1}, \quad n=2,3, \ldots
$$

$R \mathscr{D} \subset \mathscr{D}$ implies $\Phi_{n} \in \mathscr{D}$, and it is obvious from (1) that $\lim _{n \rightarrow \infty} \Phi_{n}=0$. Next, taking into account that $F_{n} \leqq E_{n}=E\left(\Delta_{n}\right), \Delta_{n}=\left(\alpha_{n}, \alpha_{n}+1\right)$, the intervals $\Delta_{n}$ and $\Delta_{m}$ do not intersect if $m \neq n$ and $E_{n}$ commutes with $\tilde{A}$, we get

$$
F_{k} \tilde{A} F_{n}=\delta_{k n} F_{n} \tilde{A} F_{n} .
$$

Due to $T_{n}^{*}=T_{n}^{*} F_{n}$ and $\Phi_{n}=F_{n} \Phi_{n}$, this gives us

$$
X \Phi_{n}=\frac{1}{\sqrt{\alpha_{n}}} T_{n}^{*} A \Phi_{n} .
$$


Whence we obtain

$$
\begin{aligned}
\left\|\Phi_{1}-X \Phi_{n}\right\| & =\left\|T_{n}^{*}\left(\sqrt{\alpha_{n}} \Phi_{n}-\frac{1}{\sqrt{\alpha_{n}}} A \Phi_{n}\right)\right\| \\
& \leqq \frac{1}{\sqrt{\alpha_{n}}}\left\|T_{n}^{*}\right\| \cdot\left\|A \Phi_{n}-\alpha_{n} \Phi_{n}\right\| \leqq \frac{1}{\sqrt{\alpha_{n}}}\left\|\Phi_{n}\right\| \underset{n \rightarrow \infty}{\longrightarrow} 0
\end{aligned}
$$

[the last inequality follows from the spectral theorem due to $\Phi_{n} \in E\left(\Delta_{n}\right) \mathscr{H}$ ]. It means that $\lim _{n \rightarrow \infty} X \Phi_{n}=\Phi_{1} \neq 0$. On the other hand, if $R \mathscr{D} \subset \mathscr{D}$, then it follows from $B \in R, A \in \mathscr{P}$ that $X^{*}=A^{*} B^{*}$ has a dense domain and so $X$ is closable. This contradiction proves that $R$ is not a type I or III factor.

Now, let us consider the case $R=\bigoplus_{i=1}^{n} R_{i}$, where $R_{i}$ are type I or III factors. Let us show that $P_{i} \in \mathscr{P}_{s}^{\prime}, P_{i}$ being projections on the subspaces $\mathscr{H}_{i}$ of the factors $R_{i}$. In both cases under consideration now $\left(\mathscr{P}_{s}^{\prime}=\mathscr{P}_{w}^{\prime}\right.$ or $\mathscr{P}$ is generated by a system of essentially self-adjoint operators) $\mathscr{P}_{w}^{\prime}$ is a von Neumann algebra: in the first case it is obvious and in the second one it follows from Proposition 1.1 in [4, p. II]. So we have

$$
P_{i} \in R \cap R^{\prime} \subset R \cap \mathscr{P}_{w}^{\prime} \subset \mathscr{P}_{s}^{\prime},
$$

the last inclusion being implied by $R \mathscr{D} \subset \mathscr{D}$. It is easy to check (cf. Proposition 3.1 in $[4, \mathrm{p} . \mathrm{II}])$ that if $P_{i} \in \mathscr{P}_{s}^{\prime}$, then the restriction $\mathscr{P}_{i}=\mathscr{P}_{\mathscr{D}_{i}}, \mathscr{D}_{i}=P_{i} \mathscr{D}$, is an Op*-algebra in $\mathscr{H}_{i}$ with the domain $\mathscr{D}_{i}$; moreover, $R \mathscr{D} \subset \mathscr{D}$ implies $R_{i} \mathscr{D}_{i} \subset \mathscr{D}_{i}$ and due to $n<\infty$, at least one of $\mathscr{P}_{i}$ is nontrivial. The problem will be reduced completely to that considered above if we prove

Lemma. Let $(\mathscr{P}, \mathscr{D})$ be an $\mathrm{Op}^{*}$-algebra, $P_{1}$ a projection from $\mathscr{P}_{s}^{\prime}, \mathscr{D}_{1}=P_{1} \mathscr{D}$, $\mathscr{P}_{1}=\left.\mathscr{P}\right|_{\mathscr{D}}$. Then

1) if $R$ and $\mathscr{P}_{w}^{\prime}$ are von Neumann algebras, then $R \supset\left(\mathscr{P}_{w}^{\prime}\right)^{\prime}$ implies $R_{P_{1}} \supset\left(\mathscr{P}_{1, w}^{\prime}\right)^{\prime}$;

2) $\mathscr{P}_{s}^{\prime}=\mathscr{P}_{w}^{\prime}$ implies $\mathscr{P}_{1, s}^{\prime}=\mathscr{P}_{1, w}^{\prime}$; is $\mathscr{P}_{1}$.

3) if $\mathscr{P}$ is generated by a system of essentially self-adjoint operators, then so

Proof of the Lemma. The first property follows from the equality proved in Proposition 3.4 of $[4, \mathrm{p} . \mathrm{I}]$ :

$$
\mathscr{P}_{1, w}^{\prime}=\left(\mathscr{P}_{w}^{\prime}\right)_{P_{1}}
$$

(in [4] $P_{1}$ was supposed to be cyclic but this was not used in the proof of (4)). To get the second property, let us notice that if $\mathscr{P}_{s}^{\prime}=\mathscr{P}_{w}^{\prime}$, then an arbitrary element of the von Neumann algebra $\left(\mathscr{P}_{s}^{\prime}\right)_{P_{1}}$ is of the form $\left.P_{1} B\right|_{P_{1} \mathscr{H}}$ with $B \in \mathscr{P}_{s}^{\prime}$. Taking into account $\mathscr{P}\left(P_{1} \mathscr{D}\right) \subset P_{1} \mathscr{D}$, we have for all $\Phi \in \mathscr{D}, A \in \mathscr{P}$ :

$$
\begin{aligned}
\left.\left.P_{1} B\right|_{P_{1} \mathscr{H}} A\right|_{P_{1} \mathscr{D}}\left(P_{1} \Phi\right) & =P_{1} B A P_{1} \Phi=A P_{1} B P_{1} \Phi \\
& =\left.\left.A\right|_{P_{1} \mathscr{D}} P_{1} B\right|_{P_{1} \mathscr{H}}\left(P_{1} \Phi\right),
\end{aligned}
$$

which means $\left(\mathscr{P}_{s}^{\prime}\right)_{P_{1}} \subset \mathscr{P}_{1, s}^{\prime}$, and due to (4) this gives us the desired:

$$
\mathscr{P}_{1, s}^{\prime} \subset \mathscr{P}_{1, w}^{\prime}=\left(\mathscr{P}_{s}\right)_{P_{1}} \subset \mathscr{P}_{1, s}^{\prime} .
$$

Finally, we shall show that if $A \in \mathscr{P}$ is essentially self-adjoint then so is $A_{1}=\left.A\right|_{P_{1} \mathscr{D}} \in \mathscr{P}_{1}$. From $P_{1} A \subset A P_{1}$ we have $P_{1} \bar{A} \subset \bar{A} P_{1}$, which implies that 
$P_{1} \operatorname{dom} \bar{A} \subset \operatorname{dom} \bar{A}$ and the restriction $\left.A\right|_{P_{1} \operatorname{dom} \bar{A}}$ is well-defined. It is easy to see that it is a self-adjoint operator for it obviously is closed and has the dense (in $P_{1} \mathscr{H}$ ) set of analytical vectors of the form $P_{1} \chi, \chi$ being analytical vectors of $\bar{A}$. Let us show that

$$
\left.\bar{A}\right|_{P_{1} \operatorname{dom} \bar{A}} \subset \bar{A}_{1} .
$$

For every $\Phi \in P_{1} \operatorname{dom} \bar{A}$ we have $\Phi=P_{1} \chi, \chi \in \operatorname{dom} \bar{A}$ and $\chi=\lim _{n \rightarrow \infty} \chi_{n}$, where $\left\{\chi_{n}\right\}$ is a sequence of vectors from $\mathscr{D}$ such that $\lim _{n \rightarrow \infty} A \chi_{n}=\bar{A} \chi$. Whence it follows that the sequence $\Phi_{n}=P_{1} \chi_{n}$ converges to $\Phi$ and the sequence $A_{1} \Phi_{n}=P_{1} A \chi_{n}$ is also convergent due to the convergence of $A \chi_{n}$. It implies $\Phi \in \operatorname{dom} \bar{A}_{1}$, and so (5) is proved. Now, if $\left.\bar{A}\right|_{P_{1} \operatorname{dom} \bar{A}}$ is self-adjoint, then so is $\bar{A}_{1}$. q.e.d.

Turning back to the proof of the theorem, we see that in some of the subspaces $\mathscr{H}_{i}$ there are nontrivial Op*-algebra $\mathscr{P}_{i}$ and von Neumann algebra $R_{i}$ satisfying all the conditions of the first part of the theorem which was already proved. It means that $R_{i}$ is not a type I or type III factor and the theorem is proved.

The idea of the proof was first suggested in [5], where the result was obtained close to the $E W^{*}$ part of the following

Corollary 1. Let $\mathscr{P}$ be a closed $E W^{*}$-algebra [6] or a $S V^{*}$-algebra [7] generated by a system of essentially self-adjoint operators or such that $\mathscr{P}_{s}^{\prime}=\mathscr{P}_{w}^{\prime}$. Then the algebra $\mathscr{P}_{b}$ is not a type I factor or type III factor or finite direct sum of such factors. If $\mathscr{P}$ is a $S V^{*}$-algebra including at least one unbounded essentially self-adjoint operator, then $\mathscr{P}_{b}$ is not a type I or type III factor.

Proof. For the listed classes of Op*-algebras, $\mathscr{P}_{b}=\left(\mathscr{P}_{w}^{\prime}\right)^{\prime}$; moreover, for a closed $E W^{*}$-algebra, $\mathscr{P}_{s}^{\prime}=\mathscr{P}_{w}^{\prime}$.

A similar fact takes place in the theory of Baire $O^{*}$-algebras $E$ : their bounded parts $E_{b}$ are $A W^{*}$-algebras and it was proved recently [8] that if $E=E_{b}$, then $E_{b}$ is not an $A W^{*}$-factor of type Ior type III and is not a finite direct sum of such factors.

Now, let us turn to physical implications. It is clear that the type I part of the theorem puts some restrictions on the global structure and the type III part on the local structure of nets of $W^{*}$ field algebras.

Corollary 2. Let a Wightman theory be given in which the field $\mathrm{Op}^{*}$-algebra $(\mathscr{P}, \mathscr{D})$ is such that $\mathscr{P}_{s}^{\prime}=\mathscr{P}_{w}^{\prime}$ or is generated by a system of essentially self-adjoint field operators. Let the global $W^{*}$ field algebra $\mathscr{F}=\left(\mathscr{P}_{w}^{\prime}\right)^{\prime}$ be such that $\mathscr{F} \mathscr{D} \subset \mathscr{D}$. Then

1) $\mathscr{F}$ is not a direct sum of type I factors;

2) if there is a strongly cyclic vacuum vector, then there is the infinite vacuum degeneration, i.e. $\operatorname{dim} \mathscr{H}_{0}=\infty, \mathscr{H}_{0}$ being the subspace of all translationally invariant vectors.

Proof. By virtue of the theorem, $\mathscr{F}$ is not a type I factor or finite direct sum of such factors. Infinite sum is now excluded, too: the reduction of the Wightman theory to the subspace $\mathscr{H}_{i}$ of any member of this sum is again a certain Wightman theory, and so the corresponding field $\mathrm{Op}^{*}$-algebra must be nontrivial. Validity of the theorem for the algebras $\mathscr{P}_{i}$ and $\mathscr{F}_{i}$ is shown in the lemma. Now, if the theory has a strongly cyclic vacuum vector, but at the same time $\operatorname{dim} \mathscr{H}_{0}<\infty$, then due to 
Theorem 2.5 in $[4$, p. II $], \mathscr{F}$ is a finite direct sum of type I factors which contradicts the theorem.

It makes sense to formulate the inverse statement as well.

Corollary 3. Let a Wightman theory be given in which the field Op*-algebra $(\mathscr{P}, \mathscr{D})$ is such that $\mathscr{P}_{s}^{\prime}=\mathscr{P}_{w}^{\prime}$ or is generated by a system of essentially self-adjoint field operators. Let this theory possess the normal global structure, i.e. $\mathscr{F}=\left(\mathscr{P}_{w}^{\prime}\right)^{\prime}$ is a direct sum of discrete factors. Then

1) $\mathscr{F} \mathscr{D} \varangle \mathscr{D}$;

2) $\mathscr{P}_{b} \varsubsetneqq \mathscr{F}$;

3) $\mathscr{P}$ is not a $S V^{*}$-algebra or closed $E W^{*}$-algebra.

The implications for local algebras $\mathscr{F}(\mathcal{O})$ are obvious: in all cases when these algebras are proved to be type III factors (and the conditions on the field Op*algebra are met) they cannot leave the domain of field operators invariant. Indeed, for any open region $\mathcal{O}$ in the Minkowski space $M$, the field operators $A(f)$, supp $f \subset \mathcal{O}$, generate the Op*-algebra $\mathscr{P}(\mathcal{O}) \subset \mathscr{P}$ with the domain $\mathscr{D}(\mathcal{O})=\mathscr{D}$. Von Neumann field algebras in theories with essentially self-adjoint field operators $A(f), f=\bar{f} \in S\left(R^{4}\right)$ are defined by the formula

$$
\mathscr{F}(\mathcal{O})=\{\exp [i \overline{A(f)}] \mid \operatorname{supp} f \subset \mathcal{O}\}^{\prime \prime}=\left(\mathscr{P}(\mathcal{O})_{w}^{\prime}\right)^{\prime}
$$

(the second equality can easily be checked, cf. Proposition 1.1 in [4, p. II]) and the definition $\mathscr{F}(\mathcal{O})=\left(\mathscr{P}(\mathcal{O})_{w}^{\prime}\right)^{\prime}$ can be preserved in general theory. Besides this, in general theory some other definitions are used, in particular [9],

$$
\hat{\mathscr{F}}(\mathcal{O})=\left\{T \in \mathfrak{B}(\mathscr{H}) \mid T \overline{A(f)} \subset \overline{A(f)} T, T A(f)^{*} \subset A(f)^{*} T, \operatorname{supp} f \subset \mathcal{O}^{\prime}\right\} .
$$

Type III for local algebras is proved in general theory (with the unique vacuum vector $\Omega$ ) only for some classes of unbounded regions, of which the most interesting are wedge regions

$$
W_{R}=\left\{x \in M\left|x^{1}>\right| x^{0} \mid\right\} ; \quad W_{L}=\left\{x \in M\left|x^{1}<-\right| x^{0} \mid\right\} .
$$

According to [9] and [1, Corollary 2 to Theorem 8.7], if $\Omega$ is cyclic for $\hat{\mathscr{F}}\left(W_{R}\right)$, then

$$
\mathscr{F}\left(W_{R}\right)=\hat{\mathscr{F}}\left(W_{R}\right)=\mathscr{P}\left(W_{L}\right)_{w}^{\prime} .
$$

Taking this into account, we obtain

Corollary 4. Let a Wightman theory be given in which the vacuum vector is unique and cyclic for $\hat{\mathscr{F}}\left(W_{R}\right)$ and the Op*-algebra $\mathscr{P}\left(W_{R}\right)$ is such that $\mathscr{P}\left(W_{R}\right)_{s}^{\prime}=\mathscr{P}\left(W_{R}\right)_{w}^{\prime}$ or has at least one essentially self-adjoint operator. Then

1) $\mathscr{F}\left(W_{R}\right) \mathscr{D} \varangle \mathscr{D}$;

2) $\mathscr{P}\left(W_{R}\right)_{b} \varsubsetneqq \mathscr{F}\left(W_{R}\right)$;

3) $\mathscr{P}\left(W_{R}\right)$ is not a $S V^{*}$ or closed $E W^{*}$-algebra.

Finally, as we know, in theories of free fields of arbitrary spins and masses, the $W^{*}$ field algebras $\mathscr{F}(D)$ of double cones $D=D^{\prime \prime}$ are generated by essentially selfadjoint field operators and are type I or III factors in the fermion theories and type III factors in the boson ones. So we have 
Corollary 5. Let $\{\mathscr{F}(\mathcal{O})\}$ be a net of $W^{*}$ field algebras of an arbitrary free field. Then for any four-dimensional open region $(\cap \subset M$,

1) $\mathscr{F}(\mathcal{O}) \mathscr{D} \varangle \mathscr{D}$;

2) $\mathscr{P}(\mathcal{O})_{b} \varsubsetneqq \mathscr{F}(\mathcal{O})$;

3) $\mathscr{P}(\mathcal{O})$ is not a $S V^{*}$ - or closed $E W^{*}$-algebra.

Clearly, this list of implications can be continued if other results on types of global and local field algebras are available.

\section{References}

1. Horuzhy, S.S.: Introduction to algebraic quantum field theory. Moscow: Nauka 1985 (in Russian)

2. Dixmier, J.: Les $C^{*}$-algèbres et leurs représentations. Paris: Gauthier-Villars 1969

3. Dixmier, J.: Les algèbres d'opérateurs dans l'éspace Hilbertien (les algèbres de von Neumann). Paris: Gauthier-Villars 1969

4. Voronin, A.V., Sushko, V.N., Horuzhy, S.S.: Algebras of unbounded operators and vacuum superselection rules in quantum field theory. I. Theor. Math. Phys. 59, 28-48 (1984); II. Theor. Math. Phys. 60, 323-343 (1984) (in Russian)

5. Kröger, P.: On EC*-algebras. Preprint Karl-Marx Universität, Leipzig (1976)

6. Inoue, A.: On a class of unbounded operator algebras. Pacific J. Math. 65, 77-96 (1977)

7. Epifanio, G., Trapani, C.: $V^{*}$-algebras: A particular class of unbounded operator algebras. $\dot{J}$. Math. Phys. 25, 2633-2637 (1984)

8. Chilin, V.I.: Baire ordered *-algebras. DAN SSSR ser. math. 258, 1065-1069 (1981) (in Russian)

9. Bisognano, J.J., Wichmann, E.: On the duality condition for quantum fields. J. Math. Phys. 17, 303-321 (1976)

Communicated by R. Haag

Received August 1, 1985 